\title{
Fast Time-varying modal parameter identification algorithm based on two-layer linear neural network learning for subspace tracking*
}

\author{
Kai Yang \\ Department of Astronautical Science and Mechanics, Harbin Institute of Technology, Harbin, Heilongjiang, China \\ E_mail:yg.hit@hotmail.com \\ Kaiping $\mathrm{Yu}$ \\ Department of Astronautical Science and Mechanics, Harbin Institute of Technology, Harbin, Heilongjiang, China \\ E_mail:yukp@hit.edu.cn
}

\begin{abstract}
The key of fast identification algorithm of time-varying modal parameter based on subspace tracking is to find efficient and fast subspace-tracking algorithm. This paper presents a modified version of NIC(Novel Information Criterion) adopted in two-layer linear neural network learning for subspace tracking, which is applied in time-varying modal parameter identification algorithm based on subspace tracking and get a new time-varying modal parameter identification algorithm. Comparing with the original subspace-tracking algorithm, there is no need to set a key control parameter in advance. Simulation experiments show that new time-varying modal parameter identification algorithm has a faster convergence in the initial period and a real experiment under laboratory conditions confirms further its validity of the time-varying modal identification algorithm presented in this paper.
\end{abstract}

Index Terms - subspace tracking, time-varying modal parameter, identification algorithm, neural network learning

\section{Introduction}

Linear time-varying (LTV) structures are widely existed in the field of aerospace, mechanics and transportation, such as expending of solar panels and mechanical arms and high-speed train ${ }^{[1]}$. For linear timeinvariable(LTI) system, measuring and analyzing techniques of modal parameters have reached a mature development. However the conventional concepts of modal parameters are out of invalidation for LTV. By adopting definition of modal parameters in LTI and using "time frozen" technique, the concept of "pseudo model parameters" ${ }^{2,3]}$ is proposed. Liu ${ }^{[4,5]}$ extended identification algorithms of modal parameters based on subspace in LTV. The procedure of modal parameter identification algorithm based on subspace is that: first extract signal subspace by applying input/output timeserials, then estimate system matrix, finally obtain timevarying modal parameters by modal theory. $\mathrm{Yu}^{[6]}$ solved time-varying modal parameter identification of moving mass/simple-supported beam by using modal parameter identification algorithm based on subspace of ensemble data $^{[2]}$. Pang ${ }^{[7]}$ got a new version of the algorithm based on subspace of ensemble data by replacing input matrix with generalized observability matrix and applying orthogonality of singular matrix, whose compute load and noise immunity are both better than that of the original one. But the above-mentioned methods are is not suitable for tracking modal parameters for large compute load and memory space. So identification algorithms based on recursive subspace derived from batch subspace method $^{[8]}$ takes the advantage in on-line modal parameter identification. F. Tasker et. $\mathrm{al}^{[9,10]}$ proposed on-line identification algorithm of time-varying modal parameters using TQR-SVD ${ }^{[11]}$ for tracking signal subspace. $\mathrm{Wu}^{[12]}$ obtained a new on-line algorithm by introducing FAST(Fast Approximate Subspace Tracking) and applied in time-varying modal parameter identification of three-link system. Verhaegen and $\mathrm{Yu}^{[13]}$ developed a class of subspace model identification algorithms to identify periodically and arbitrarily timevarying system using a single input-output data batch, which was different from the algorithm referred in Ref.[2] and [7]. Oku and Kimura ${ }^{[14]}$ proposed recursive 4SID algorithms based on gradient type subspace tracking. Pang et. al $^{[15,16]}$ got a fast identification algorithm of time-varying modal parameters by introducing PAST $^{[17]}$ (Projection Approximation Subspace Tracking) and applied in two-link system and moving mass/simplesupported beam system.

The key of algorithms based on subspace tracking is to find an efficient and fast algorithm for subspace tracking. The signal subspace obtained by PAST converges asymptotically to the orthogonal subspace, by introducing orthogonal method $W=W\left(W^{T} W\right)^{-1 / 2}$ OPAST $^{[18]}$ is obtained. Otherwise by deflation technique of PCA(Principal Component Analysis), PASTd ${ }^{[17,19]}$ is derived. Similarly, signal subspace obtained by PASTd has a strong lose of orthogonality, by introducing orthogonal method Gram-Schmit for incorporating

\footnotetext{
* This work is supported by NSF Grant \#10672045 to Yu Kaiping
} 
characteristic of PASTd, a modified PASTd ${ }^{[20]}$ is obtained. PAST can be viewed as a classical power iteration method. Comparing with PAST, NPI ${ }^{[21,22]}$ (Natural Power Iteration) has a faster convergence speed and ensures orthogonality of subspace vectors without compute load increasing. In both PAST and NPI, $W_{p+1} \approx W_{p}$ is used in the process of algorithm derivation, so they are only suitable for tracking slow subspace. Different from PAST and NPI, $\mathrm{API}^{[23]}$ (Approximated Power Iteration) can track rapid subspace and apply updating data in both infinite exponential window and finite moving exponential window. Different from PAST in cost function, $\mathrm{NIC}^{[24,25]}$ (Novel Information Criterion) is proposed, adopting in two-layer linear neural network learning for subspace tracking. By introducing orthogonal method, a modified version is obtained named FONIC ${ }^{[26]}$.

This paper presents a modified version of NIC adopting in two-layer linear neural network learning for subspace tracking. Comparing with the original algorithm, there is no need to set an important parameter in advance. Simulation experiments show that it has a faster convergence in the initial period.

\section{Modal parameter identification algorithm based on ED/SVD}

The state-space model of linear time-invariable system of $n$ / 2 order is denoted as follows:

$$
\begin{aligned}
& x(k+1)=A x(x)+B u(k), \\
& y(k)=C x(k)+D u(k)
\end{aligned}
$$

Where $x(k) \in R^{n \times 1}$ is the state vector at the $k^{\text {th }}$ sampling instant, $A \in R^{n \times n}$ is the system state matrix, $B \in R^{n \times m}$ is the input matrix, $u(k) \in R^{m \times 1}$ is the corresponding input vector at the $k^{\text {th }}$ sampling instant, $C \in R^{r \times n}$ is the input matrix , $D \in R^{r \times m}, y(k)$ the output vector.

Constructing Hankel matrix by input/output vectors respectively, we have:

$$
\begin{gathered}
U_{p}=\left[\begin{array}{cccc}
u(1) & u(2) & \cdots & u(p) \\
u(2) & u(3) & \cdots & u(p+1) \\
\vdots & \cdots & \ddots & \vdots \\
u(M) & u(M+1) & \cdots & u(p+M-1)
\end{array}\right] \\
Y_{p}=\left[\begin{array}{cccc}
y(1) & y(2) & \cdots & y(p) \\
y(2) & y(3) & \cdots & y(p+1) \\
\vdots & \vdots & \ddots & \vdots \\
y(M) & y(M+1) & \cdots & y(p+M-1)
\end{array}\right]
\end{gathered}
$$

Further we get

$$
Y_{p} U_{p}^{\perp}=Y_{p}-Y_{p} U_{p}^{T}\left(U_{p} U_{p}^{T}\right)^{-1} U_{p}=\Gamma X U_{p}^{\perp}
$$

Where $U_{P}^{\perp}=I-U_{p}^{T}\left(U_{p} U_{p}^{T}\right)^{-1} U_{p}, \quad I$ is an identity matrix, $\Gamma=\left[\begin{array}{llll}C & C A & \cdots & C A^{M-1}\end{array}\right]^{T}$ is the generalized observability
$X=\left[\begin{array}{llll}x(1) & x(2) & \cdots & x(p)\end{array}\right]$ is a matrix constituted by state vector, superscript $(\square)^{T}$ denotes matrix transpose.

Do singular value decomposition(SVD) on $Y_{p} U_{p}^{\perp}$, we have:

$$
Y_{p} U_{p}^{\perp}=\left[\begin{array}{ll}
P_{s} & P_{n}
\end{array}\right]\left[\begin{array}{cc}
\Sigma_{s} & 0 \\
0 & \Sigma_{n}
\end{array}\right]\left[\begin{array}{c}
V_{s}^{T} \\
V_{n}^{T}
\end{array}\right]
$$

Where $P_{s}$ is estimate of $\Gamma$. For $Y_{p} U_{p}^{\perp}\left(Y_{p} U_{p}^{\perp}\right)^{T}=Y_{p} U_{p}^{\perp} Y_{p}^{T}$, eigen-decomposition(ED) on $Y_{p} U_{p}^{\perp} Y_{p}^{T}$ can be used to extract left-singular matrix block $P_{s}$ of $Y_{p} U_{p}^{\perp}$. By (4), we have:

$$
A=\Gamma_{1}^{+} \Gamma_{2}
$$

Where $\Gamma_{1}$ and $\Gamma_{2}$ are the first $M-1$ block row and last $M-1$ block row respectively. Superscript $(\square)^{+}$ denotes Moore-Penrose generalized matrix inverse. Doing eigen-decomposition on $A$, we have:

$$
A=\Phi \Lambda \Phi^{T}
$$

Where $\quad \Lambda=\operatorname{diag}\left(\begin{array}{llll}\lambda_{1} & \lambda_{2} & \cdots & \lambda_{2 n}\end{array}\right) \quad$ and $\lambda_{i}=\lambda_{i+n}^{*}$, superscript $*$ denotes conjugate. By modal analysis theory, we get:

$$
\omega_{i}=\sqrt{\left(\lambda_{i}^{R}\right)^{2}+\left(\lambda_{i}^{I}\right)^{2}}, \xi_{i}=-\lambda_{i}^{R} / \omega_{i}
$$

Where $\omega_{i}$ and $\xi_{i}$ are the natural frequency and damping ratio respectively of the $i^{\text {th }}$ order, $\lambda_{i}^{R}$ and $\lambda_{i}^{I}$ are the real and imaginary parts respectively of eigenvalue $\lambda_{i}$. In general, natural frequency and damping ratio are included in modal parameters. Here in this paper we focus on time-varying frequency identification.

\section{Pre-treatment on the updating input/output data}

From the second part, evidently each step eigendecomposition on $Y_{p} U_{p}^{\perp} Y_{p}^{T}$ costs a large memory space and computation load. Otherwise there is no need to evaluate $Y_{p} U_{p}^{\perp} Y_{p}^{T}$ and do eigen-decomposition on $Y_{p} U_{p}^{\perp} Y_{p}^{T}$ for extracting its eigen-matrix in the timevarying modal parameter identification algorithm based on subspace tracking. By adopting recursive form of updating input/output data, principle subspace tracking algorithm can be applied and then fast identification of time-varying modal parameter is achieved. Updating the input and output Hankel matrix, we have: 


$$
Y_{p+1}=\left[\begin{array}{ll}
Y_{p} & \bar{y}_{p+1}
\end{array}\right], U_{p+1}=\left[\begin{array}{ll}
U_{p} & \bar{u}_{p+1}
\end{array}\right]
$$

Where

$$
\begin{aligned}
& \bar{y}_{p+1}=\left[\begin{array}{llll}
y(p+1) & y(p+2) & \cdots & y(p+M)
\end{array}\right]^{T}, \\
& \bar{u}_{p+1}=\left[\begin{array}{llll}
u(p+1) & u(p+2) & \cdots & u(p+M)
\end{array}\right]^{T} .
\end{aligned}
$$

By lemma of matrix inverse

$$
\left(A+x x^{T}\right)^{-1}=A^{-1}-A^{-1} x x^{T} A^{-1} /\left(1+x^{T} A^{-1} x\right)
$$

We have:

$$
Y_{p+1} U_{p+1}^{\perp} Y_{p}^{T}=Y_{p} U_{p}^{\perp} Y_{p}^{T}+z_{p+1} z_{p+1}^{T}
$$

Where $Z_{p+1}=\left[Y_{p} U_{p}^{T}\left(U_{p} U_{p}^{T}\right)^{-1} \bar{u}_{p+1}-\bar{y}_{p+1}\right] / \sqrt{1+\alpha_{p+1}}$, $\alpha_{p+1}=\bar{u}_{p+1}^{T}\left(U_{p} U_{p}^{T}\right)^{-1} \bar{u}_{p+1}$. Equation (11) is called rank-one updating. If there is no persistent excitation, namely exciting by initial displacement or velocity conditions, then we have $z_{p+1}=\bar{y}_{p+1}, U_{p}^{\perp} \in C^{p \times p}$ identity matrix. Such condition is common in the engineering field. Both are referred in this paper.

\section{Time-varying modal parameter identification} algorithm based on neural network learning

After getting the recursive form of updating input/output data, the key of fast identification of timevarying modal parameters is efficient and fast algorithm for subspace tracking. The following is to state the algorithm based on linear two-layer neural network for subspace tracking.

The cost function ${ }^{[23,24]}$ is chosen as:

$$
J_{N I C}\left(W_{k}\right)=\frac{1}{2}\left\{\operatorname{tr}\left[\log \left(W_{k}^{T} R_{z}(k) W_{k}\right)\right]-\operatorname{tr}\left(W_{k}^{T} W_{k}\right)\right\}
$$

Where $W_{k}$ is the signal subspace at $k^{\text {th }}$ instant.

$R_{z z}(k)=\sum_{i=1}^{k} \beta^{k-i} z_{i} z_{i}^{T}, 0<\beta<1$ is the forgetting factor,

$\operatorname{tr}(\square)$ denotes matrix trace.

By partial differential of matrix trace, we have:

$$
\nabla J_{N I C}\left(W_{k}\right)=R_{z z}(k) W_{k}\left(W_{k}^{T} R_{z z}(k) W_{k}\right)^{-1}-W_{k}
$$

Further we have:

$$
W_{k}=W_{k-1}+\eta_{k} \nabla J_{N I C}\left(W_{k}\right)
$$

$$
W_{k}=\left(1-\eta_{k}\right) W_{k-1}+\eta_{k} R_{\mathbb{Z}}(k) W_{k}\left(W_{k}^{T} R_{\mathbb{Z}}(k) W_{k}\right)^{-1}
$$

Let $y_{k}=W_{k-1} z_{k}$, we get,

$$
W_{k}=\left(1-\eta_{k}\right) W_{k-1}+\eta_{k} R_{z y}(k) R_{y y}^{-1}(k)
$$

Where $R_{z y}(k)=\sum_{i=1}^{k} \beta^{k-i} z_{i} y_{i}^{T}, R_{t y}(k)=\sum_{i=1}^{k} \beta^{k-i} y_{i} y_{i}^{T}$.

By lemma of matrix inverse,

$$
\begin{array}{r}
\left(A+z z^{T}\right)^{-1}=A^{-1}-A^{-1} z z^{T} A^{-1} /\left(1+z^{T} A^{-1} z\right), \text { We get } \\
W_{k}=\left(1-\eta_{k}\right) W_{k-1}+\eta_{k} \bar{W}_{k}
\end{array}
$$

Where,

$$
\begin{aligned}
& \bar{W}_{k}=\bar{W}_{k-1}+\left(z_{k}-\bar{W}_{k-1} y_{k}\right) y_{k}^{T} P_{k-1} /\left(\beta+y_{k}^{T} P_{k-1} y_{k}\right) \\
& , \bar{W}_{k-1}=R_{z y}(k-1) P_{k-1}, \\
& P_{k}=\left(\sum_{i=1}^{k} y_{i} y_{i}^{T}\right)^{-1}=\left(\beta R_{y y}(k-1)+y_{k} y_{k}^{T}\right)^{-1} . \\
& =\frac{1}{\beta}\left(P_{k-1}-\frac{P_{k-1} y_{k} y_{k}^{T} P_{k-1}}{\beta+y_{k}^{T} P_{k-1} y_{k}}\right)
\end{aligned}
$$

Different from the original method, $\eta_{k}$ is not set in advance. Applying Differential segment,

$$
\frac{\partial J_{N I C}\left(W_{k}\right)}{\partial \eta_{k}}=\frac{\partial J_{N I C}\left(W_{k}\right)}{\partial W_{k}} \frac{\partial W_{k}}{\partial \eta_{k}}
$$

Let $\frac{\partial J_{N I C}\left(W_{k}\right)}{\partial \eta_{k}}=0$, we have:

$$
\eta_{k}=\frac{\left\|\left(z_{k}-W_{k-1} y_{k}\right) y_{k}^{T} P_{k-1} /\left(\beta+y_{k}^{T} P_{k-1} y_{k}\right)\right\|}{\left\|\bar{W}_{k}-W_{k-1}\right\|}
$$

Where $\|$ | $\mid$ denotes Frobenius norm of matrix.

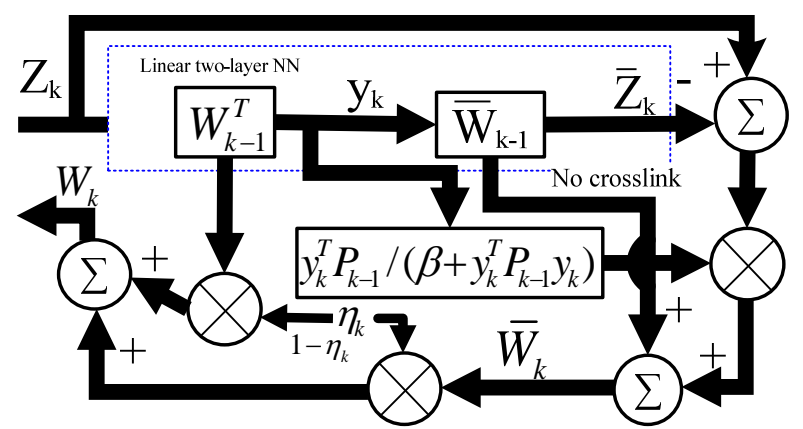

Fig. 1 Algorithm block based on linear two-layer neural network learning for subspace tracking

According to the modified version mentioned above, we get the algorithm block based on two-layer linear neural network learning for subspace tracking showed in Fig.1. Further we get the fast identification algorithm of time-varying modal parameters.

Further construct the system matrix $A(k)$ by $W_{k}$, repeating (6), (7) and (8) for extracting time-varying modal parameters. The whole procedure of the timevarying modal parameter identification algorithm based on two-layer linear neural network learning for subspace tracking is showed as follows: 


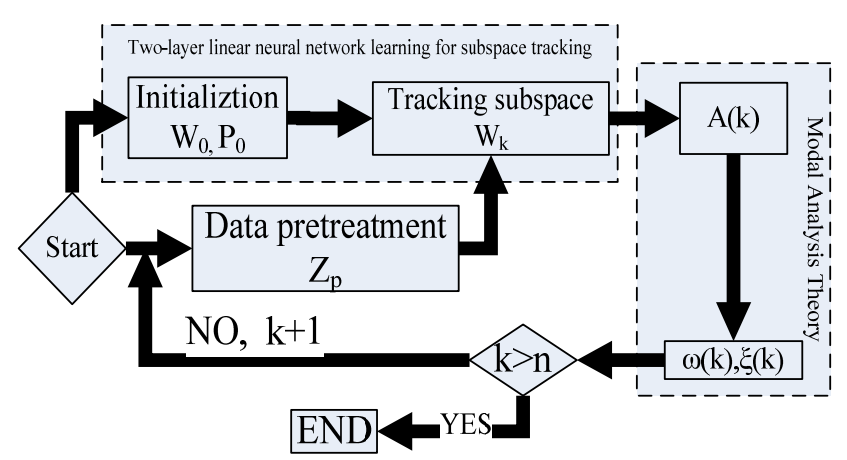

Fig.2 Flowchart of identification algorithm for time-varying modal parameters

5. Simulation and Analysis

Moving mass-beam structure is widely used in modelling bridge with moving vehicles, truss with moving crane and so on, which is a typical time-varying structure. Supposing that moving mass keeps contact with beam all the time, neglecting torsion deformation of beam, applying Euler-Bernoulli beam theory, we get the model as follows:

$$
E I \frac{\partial^{4} y(x, t)}{\partial x^{4}}+m \frac{\partial^{2} y(x, t)}{\partial t^{2}}=F(x, t)
$$

Where $E$ is Young's modulus, $I$ is the second moment of beam cross-section, $m$ is the mass per unit length of beam, $y(x, t)$ denotes the transverse deflection of beam, $F(x, t)$ is time-varying driving force. Taking the inertia load of moving mass into account ${ }^{[27]}$, we have:

$$
\begin{aligned}
& F(x, t)=\frac{1}{\varepsilon}\left[-m_{0} g-m_{0} \frac{\partial^{2} y(x, t)}{\partial t^{2}}\right]\left[H\left(x-\xi+\frac{\varepsilon}{2}\right)-\right. \\
& \left.H\left(x-\xi-\frac{\varepsilon}{2}\right)\right]-f(t) \delta(x-l)
\end{aligned}
$$

Where $m_{0}$ is the moving mass, $g$ is the gravitational acceleration, $H(\square)$ is Heaviside unit function, $f(t)$ is external excitation force, $\delta(\square)$ is Dirac-Delta function, $\xi=V t, V=$ const the moving speed of moving mass. And the other parameters are showed in Fig. 2.

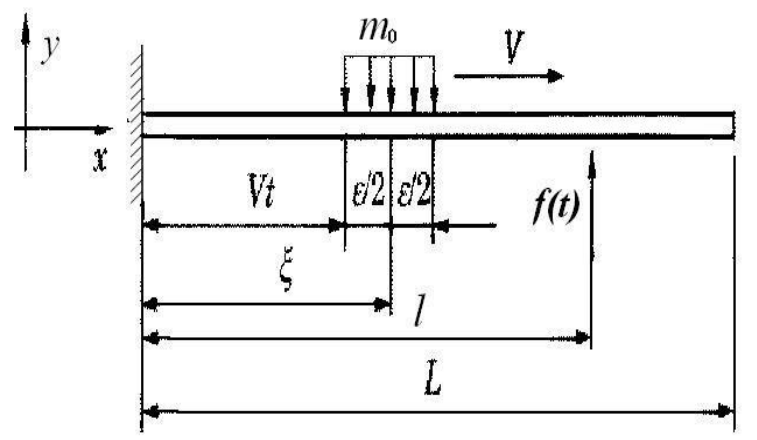

Fig.3 Moving distributed mass-Cantilever structure
Let $y(x, t)=\sum_{i=1}^{\infty} \phi_{i}(t) X_{i}(x)$, with $\phi_{i}(t)$ is

unknown functions of time and $X_{i}(x)$ is the shape functions for the $i^{\text {th }}$ mode of the vibration beam, which are chosen to be known. Substituting them into (20) and (21), we have:

Where:

$$
M(t) \Phi^{\prime \prime}(t)+\Omega^{2} \Phi(t)=\bar{F}(t)
$$

$$
\begin{aligned}
M(t)= & I_{n}+\operatorname{diag}\left(m_{i}\right) m_{0}\left[X(\xi) X^{T}(\xi)\right. \\
& +\frac{\varepsilon^{2}}{24}\left(X(\xi) X^{T}(\xi) \Lambda^{2}\right. \\
+ & \left.2 \Lambda X(\xi) X^{T}(\xi) \Lambda+\Lambda^{2} X(\xi) X^{T}(\xi)\right]
\end{aligned}
$$

$\Lambda=\operatorname{diag}\left(\beta_{i}\right)$

$\bar{X}(x)=\left[X_{1}(x) \cdots X_{i}(x) \cdots X_{n}(x)\right]^{T}$

$\Phi(t)=\left[\phi_{1}(t) \cdots \phi_{i}(t) \cdots \phi_{n}(t)\right]^{T}, I_{n} \in C^{n \times n}$ identity matrix, $\Omega=\operatorname{diag}\left(\omega_{i}\right), \operatorname{diag}(\square)$ denotes diagonal matrix, $\omega_{i}^{2}=\beta_{i}^{4} E I / m, X_{i}(x)$ should be chosen to meet that

$$
\begin{aligned}
& X_{i}(x)-\beta_{i}^{4} X_{i}^{(4)}(x)=0, m_{i}=m \int_{0}^{L} X_{i}^{2}(x) d x \\
& \bar{F}(t)=-\operatorname{diag}\left(m_{i}\right) m_{0} g\left(I_{n}+\frac{\varepsilon^{2}}{24} \Lambda^{2}\right) X(\xi)+f(t) \bar{X}(l)
\end{aligned}
$$

The dimension of beam is $1.2 \times 0.05 \times 0.008 \mathrm{~m}$, $V=0.2 \quad \mathrm{~m} / \mathrm{s}, \quad E I=418.13 \quad \mathrm{~N} . \mathrm{m}^{2}$, $\xi=0.07 \mathrm{~m}, l=1.0 \mathrm{~m}$,

$X_{i}(x)=\cosh \left(\beta_{i} x\right)-\cos \left(\beta_{i} x\right)-\frac{\cosh \left(\beta_{i}\right)+\cos \left(\beta_{i}\right)}{\sinh \left(\beta_{i}\right)+\sin \left(\beta_{i}\right)}$ $\left(\sinh \left(\beta_{i} x\right)-\sin \left(\beta_{i} x\right)\right), \beta_{i} \quad$ is determined by $1+\cos \left(\beta_{i} L\right) \cosh \left(\beta_{i} L\right)=0, i=1 \cdots n, n$ takes a limited value as $n=4$.

The identification results of time-varying modal parameter identification algorithms based on different subspace tracking methods, such as the original NIC, the modified NIC presented in this paper and FONIC, are showed in Fig. 4 and 5. The output data incorporates stochastic white noise of signal-noise ratio $\mathrm{SNR}=43 \mathrm{db}$. 

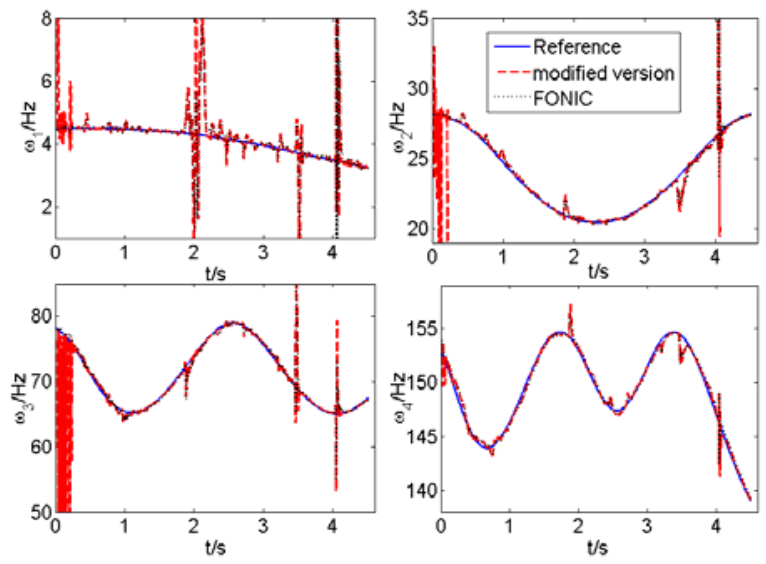

Fig. 4 Identification results with no noise disturbing
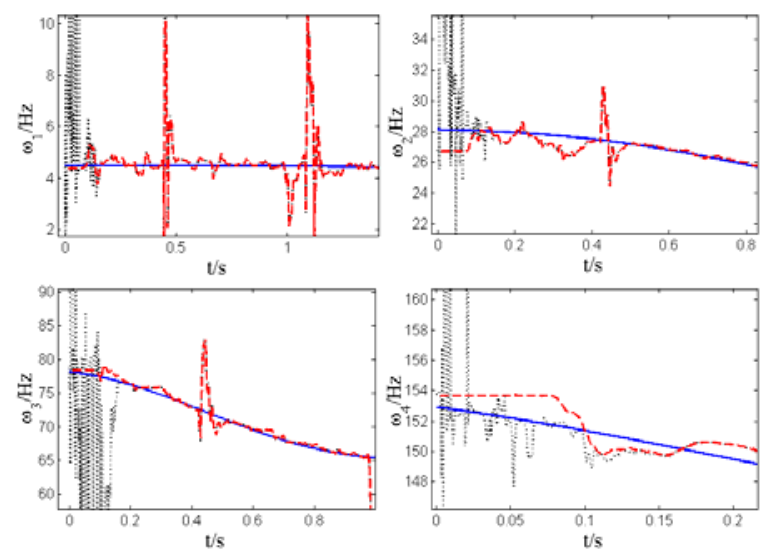

Fig. 5 Identification results with noise disturbing

By Fig.3 and Equation (22), its natural frequencies of the moving distributed mass-cantilever structure change along with time. In Fig.4, the real blue curve denotes theoretic results for reference, with the red dash curve denoting identification results obtained by applying time-varying modal parameter identification algorithm based on modified NIC,which is adopted in two-layer linear neural network learning for subspace tracking and the black dot curve denoting identification results obtained by using time-varying modal identification algorithm based on FONIC. As is shown in Fig.4, timevarying modal identification algorithm based on modified NIC has an equivalent performance with that based on FONIC. Otherwise due to adopting orthogonal method in FONIC for subspace tracking, its computation load is greater than that of modified NIC. As is showed in Fig.5, all of the three time-varying modal parameter identification algorithms based on different subspace tracking method have a good tolerance of noise. Showed In Fig.5, evidently the time-varying modal parameter algorithm based on modified NIC, which is adopted in two-layer linear neural network learning for subspace tracking, has a faster convergence speed in the initial period.

To test its performance of the time-varying modal parameter identification algorithm based on two-layer linear neural network learning for subspace tracking, a experiment of cantilever beam with removable mass is designed. Such an experiment is divided into three parts. First test its natural frequencies of cantilever beam with removable mass on it by applying conventional modal analysis method, such as Power Spectrum Density Analysis. Second repeat the first part on cantilever beam without removable mass on it. Third sample its vibration response data in the form of acceleration excited by shock and remove abruptly the moveable mass in the data sampling procedure. Do data processing by using the time-varying modal parameter identification algorithm based on two-layer linear neural network learning for subspace tracking presented in this paper. The experiment equipment is showed in Fig.6. There are two piezoelectric accelerometers, a cantilever beam, a removable mass, a hammer used as plus excitation, a set of data collecting system named DASP V10 showed in Fig. 6 and data processing system ,mainly a set of modal analysis software and corresponding hardware, doesn't be showed in Fig.6.

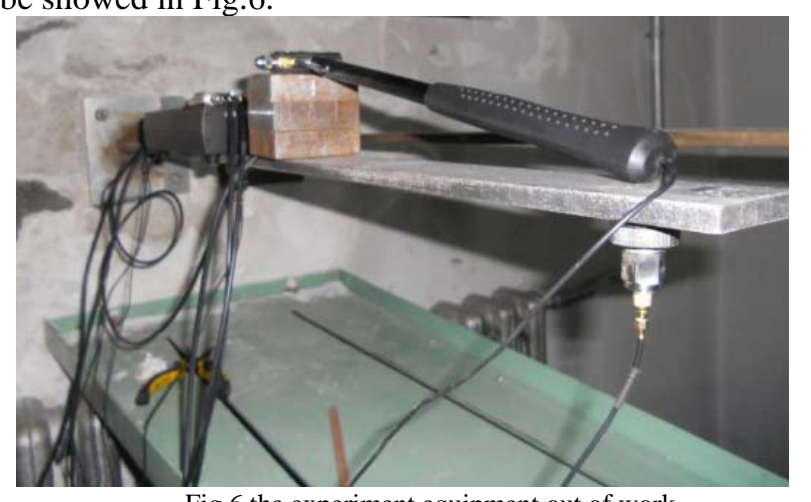

Fig.6 the experiment equipment out of work

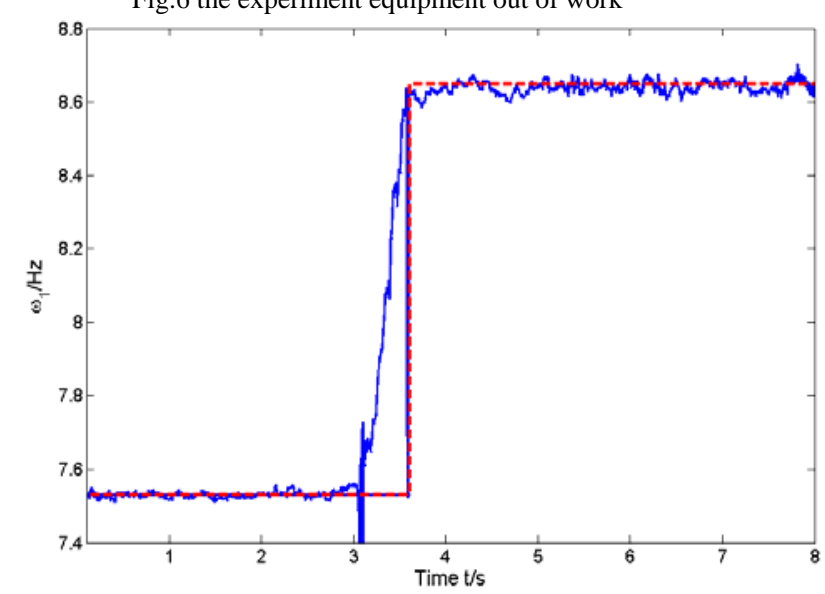

Fig.7 the first-order abrupt natural frequency 


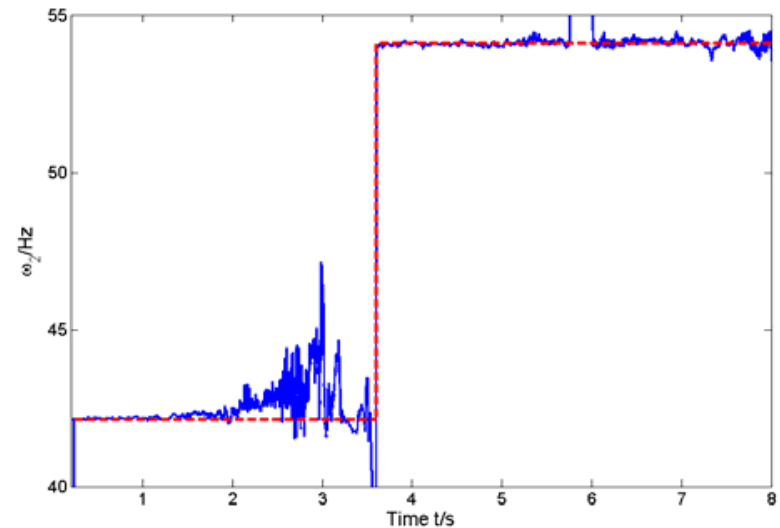

Fig.8 the second-order abrupt natural frequency

In Fig.7 and 8, the blue real curve denotes the identification results with the red dash curve denoting the testing value for reference. In Fig.7 the first-order natural frequency changes from $7.53 \mathrm{~Hz}$ to $8.65 \mathrm{~Hz}$ and in Fig. 8 the second-order natural frequency changes from $42.1 \mathrm{~Hz}$ to $54.1 \mathrm{~Hz}$. Evidently we can foresee change of natural frequencies in the third part of the designed experiment. And identification results showed in Fig. 7 and 8 just coincide with what we test, which confirms its performance of the time-varying modal parameter identification algorithm based on two-layer linear neural network learning for subspace tracking presented in this paper.

\section{Conclusion}

The key of fast identification algorithm of timevarying modal parameters is to find efficient and fast signal subspace tracking algorithms. This paper applies the modified NIC adopted in two-layer linear neural network learning for subspace tracking to achieve fast identification algorithm of time-varying modal parameters. For the modified version of NIC, using in two-layer linear neural network learning for subspace tracking, there is no need to set an important factor in advance and has a faster convergence speed in the initial period demonstrated by simulation experiment results. And real experiment under laboratory conditions confirms its validity of the time-varying modal parameter identification algorithm presented in this paper in extracting time-varying modal parameters.

\section{REFERENCE}

[1] Zou Jingxiang, Yu Kaiping and Pang Shiwei. Methods of time-varying structural parameter identification[J]. Advances in mechanics, 2000, 30(3):370-377

[2] Kefu Liu . Extension of modal analysis to linear timevarying system[J] . Journal of Sound and Vibration, 1999, 226(1):149-167

[3] Kefu Liu . Identification of linear time-varying system[J] . Journal of Sound and Vibration, 1997,206(4):487-505

[4] Juang J N and Pappa R S . An eigensystem realization algorithm for modal parameter identification and model reduction[J] . Journal of Guidance, Control and Dynamic, 1985,8(5):620-627

[5] Longman R W and Juang J N . Recursive form of the
Eigensystem Realization algorithm for system identification[J] . Journal of Guidance, Control and Dynamic, 1989, 12(5):647-652

[6] Yu Kaiping, Xie Lili, Fan Jiuming et.al . A parameter identification of simple supported beams system carrying a moving mass $[\mathrm{J}]$. Earthquake engineering and engineering vibration, 2002, 22(5):14-17

[7] Pang Shiwei, Yu Kaiping and Zou Jingxiang . Improved subspace method with application in linear tie-varying structural modal parameter indentification $[\mathrm{J}]$. Chinese Journal of applied mechanics, 2005, 22(2):184-189

[8] Yang Lifang, Yu Kaiping, Pang Shiwei et. al . Comparison study on identification methods applied to linear timevarying structures $[\mathrm{J}]$. Journal of Vibration and Shock, 2007,26(3):8-12

[9] F Tasker, A Bosse, S Fisher . Real-time modal parameter estimation using subspace methods:theory[J] . Mechanical Systems and Signal Processing,1998,12(6):797-808

[10] A Bosse, F Tasker, S Fisher . Real-time modal parameter estimation using subspace methods: application[J] . Mechanical Systems and Signal Processing, 1998, 12(6):809-823

[11] E M Dowling, L P Ammann and R D Degroat . A TQRiteration based adaptive SVD for real time angel and frequency tracking[J] . IEEE Transactions on Signal Processing, 1994,42(4):914-926

[12] Wu Riqiang, Yu Kaiping and Zou Jingxiang . An improved subspace method and its application to parameter identification of time-varying structures[J] . Engineering Mechaics，2002，19(4):67-70

[13] Michel Verhaegen , Xiaode Yu . A class of subspace model identification algorithms to identify periodically and arbitrarily time-varying systems[J] . Automatica, 1995, 31:201-216

[14] Hiroshi Oku and Hidenori Kimura . Recursive 4SID algorithms using gradient type subspace tracking[J] . Automatica, 2002,38:1035-1043

[15] Pang Shiwei, Yu Kaiping and Zou Jingxiang. A projection approximation recursive subspace method for identification of modal parameter of time-varying structures [J]. Engineering Mechanics, 2002, 22(5):115119

[16] Pang Shiwei, Yu Kaiping and Zou Jingxiang . Timevarying system identification using recursive subspace method based on free response data[J] . Journal of Vibration Engineering,2005, 18(2):233-237

[17] Bin Yang . Projection Approximation subspace tracking[J] . IEEE Transactions of Signal Processing, 1995, 43(1):95-107

[18] K Abed-Meraim, A Chkeif and Y Hua . Fast orthonormal PAST algorithm[J] . IEEE Signal Processing Letters, 2000, 7(3):60-62

[19] Bin Yang . An extension of the PASTd algorithm to both rank and subspace tracking[J] . IEEE Signal Processing Letters, 1995, 2(9):179-182

[20] Imran Ali, Doug Nyun Kim and Taikyeong Ted Jeong . A new subspace tracking algorithm using approximation of Gram-Schmidt procedure . In: 2009 International Conference on Information and Multimedia Technology, 2009, 245-248

[21] Yingbo Hua, Yong Xiang, Tianping Chen and so on . A new look at the power method for fast subspace tracking [J]. Digital Signal Processing, 1999,9:297-314

[22] Yingbo Hua, Yong Xiang, Tiangping Chen, Karim Abedmeraim et al . Natural power method for fast subspace tracking . In:Proceeding of the 1999 IEEE Signal 
Processing Society Workshop, Neural Networks for Signal Processing, 1999, 176-185

[23] Roland Badeau, Bertrand David and Gael Richard . Fast approximated power iteration subspace tracking[J] . IEEE Transactions on Signal Processing, 2005, 53(8):2391-2341

[24] Yongfeng Miao and Yingbo Hua . Fast Subspace Tracking by a Novel Information Criterion. Signals, Systems and Computer, the Thirty-First Asilomar Conference, 1997, vol2:1312-1316

[25] Yongfeng Miao and Yingbo Hua . Fast Subspace Tracking and Neural Network Learing by a Novel Information Criterion . IEEE Transactions on Signal Processing, 1998,46(7):1967-1979

[26] Amir Valizadeh, Mehdi Farrokhrooz and Ali Rafiei . Fast Signal Subspace Tracking Using Two-Layer Linear Neural Network Learning. $16^{\text {th }}$ Mediterranean Conference on Control and Automation . France, 2008:1828-1832

[27] E Esmailzadeh and M Ghorashi . Vibraton analysis of beams traversed by uniform partially distributed moving masses[J] . Journal of Sound and Vibration, 1995, 184(1):9-17

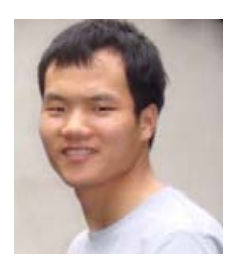

Kai Yang received the B.Sc. degree in engineering mechanics from Harbin Institute of Technology, Harbin, China in 2009 and now is pursuing for his Engineering Doctor's Degree from Harbin Institute of Technology, Harbin, China from 2009, major in General mechanics and mechanics foundation. His current research intersts are in the field of timevarying modal parameter identification, time-varying structure system modeling and modal analysis within thermodynamics.

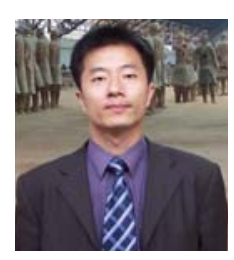

Kaiping Yu received the B.Sc. degree in engineering mechanics from Shanghai Jiao Tong University, Shanghai, China in 1989 and the M.Sc. degree in Vibration, Shock and noise and the Ph.D. degree in General mechanics and mechanics foundation from Harbin Institute of Technology, Harbin,China, in 1996 and 2000, respectively. He achieved his civil-engineering Post-doctor in Institute of engineering mechanics, China earthquake administration in 2002.He had been engaged in space station design in Department of Aircraft Design, Samara State University, Samara Oblast, Russia, from October, 1998 to March, 1999. He worked as a visiting scholar in Launch vehicle and spacecraft, Department of Special machine Manufacturing, Bauman Moscow State Technical University, Moscow, Russia from October, 2003 to October, 2004. His current research interests are computational mechanics, Structure Dynamics and control, vibration theory and its application, Dynamical theory of high-speed underwater vehicle and its trial Since 2005, he has been a professor in Department of Astronautical Science and Mechanics, Harbin Institute of Technology, Harbin, Heilongjiang, China. 